Original Research

(a) (1) \& OPEN ACCESS

\title{
Awareness of Symptoms and Early Management of Hypoglycemia among Patients with Diabetes Mellitus
}

Suresh K. Sharma', Ravi Kant ${ }^{2}$

'College of Nursing, All India Institute of Medical Sciences, Rishikesh, Uttarakhand, '2Department of General Medicine, All India Institute of Medical Sciences, Rishikesh, Uttarakhand, India

\begin{abstract}
Background: Hypoglycemia mostly occurs in diabetic patients on medications. Lack of awareness on hypoglycemic symptoms among patients may delay its identification and treatment. The symptoms of hypoglycemic symptoms among patients may delay its identification and treatment. Neglecting the symptoms of hypoglycemia and delaying treatment could cause poorer outcomes or morbidity. The present study was aimed to assess the awareness of symptoms of hypoglycemia and knowledge in early management of hypoglycemia among patients with diabetes.
\end{abstract}

Methods: A Cross sectional study was done among 500 diabetes mellitus patients attending the outpatient department of AllMS, Rishikesh, Uttarakhand in 2017. Patient with diabetes for over five years and who were on insulin treatment were included in the study. After obtaining an informed consent, knowledge on the symptoms and early treatment of hypoglycemia were collected. The data were analyzed by frequency and percentage.

Results: The study included 500 diabetic patients, of which $55.5 \%$ were females. The common symptoms of hypoglycemia known to the study subjects were dizziness (84.4\%), weakness (74.1\%), and drowsiness (68.1\%). Overall, 322 (64.4\%) diabetic patients had good knowledge on hypoglycemia (knowledge of at least three symptoms of hypoglycemia together with at least one precipitating factor and at least one remedial measure). Regarding management of hypoglycemia, $49 \%$ patients preferred taking glucose powder or sugar with water as an immediate measure. Higher age, illiteracy, low socioeconomic status was associated with poor knowledge whereas treatment with insulin along with oral hypoglycemic agents was associated with good knowledge on hypoglycemia.

Conclusion: Although the knowledge on the symptoms, remedial measures, and prevention of hypoglycemic episodes was good among the type 2 diabetic patients in the study, there were gap in knowledge on important aspects like precipitating factors, target levels etc., which need to be addressed by health care workers through regular educational programs.

Keywords: Diabetes mellitus, hypoglycemia, awareness, management, insulin.

\section{Corresponding Author:}

Dr. Suresh K. Sharma, RN, MScN, PhD.

Professor \& Principal, College of Nursing,

All India Institute of Medical Sciences,

Rishikesh, Uttarakhand, India

Email: skaiims17@gmail.com, 08475000293 
Background : The symptoms of hypoglycemia may be nonspecific with intensity decreasing with increasing age. Thus, it is very important that the subjects are able to recognize and identify the symptom onset at an early stage in order to manage the episode effectively and take steps to prevent the recurrence. Neglecting the symptoms of hypoglycemia and delaying treatment could cause poorer outcomes or morbidity.

Large trials (action to control cardiovascular risk in diabetes, Veterans affairs diabetes trial) have shown that there were was a higher mortality in the group that had hypoglycemia (intensively treated arm)., Hence, the American Diabetes Association (ADA) guidelines emphasize on individualizing targets and reducing risk of hypoglycemia in patients with long duration of diabetes and comorbidities. In a survey conducted by the American Association of Clinical Endocrinology emphasize that there was a knowledge gap which most be addressed.

In this background, the knowledge and awareness about the varied presentations of hypoglycemia and the possible early management strategies for the same would go a long way in patients with diabetes management. There is a need for shared responsibility in the prevention of hypoglycemia. We proposed to study the knowledge about hypoglycemia and its early management among diabetes patients attending AllMS, Rishikesh.

Methods : This cross sectional study study was done among purposively selected 500 diabetes mellitus patients for over five years and who were on insulin treatment attending the Diabetic Clinic of All India Institute of Medical Sciences (AllIMS), Rishikesh, Uttarakhand. Study was conducted during June 2016 to July, 2017.

Patients with diabetes mellitus-I, DM-II patients receiving only oral hypoglycemic drugs, pregnant women with gestational diabetes mellitus, diabetes secondary to other systemic diseases were excluded from the study. This study was approved by Institute Ethical Committee and after obtaining an informed written consent, patients were subjected to a semi-structured interview to assess the knowledge on the symptoms and early treatment of hypoglycemia along with base line data about the patient and disease existence.

The questions related to knowledge of the diabetic patients on the symptoms of hypoglycemia, the precipitating factors of such attacks, the immediate remedial measures which need to be adopted by them, the complications and how to prevent further episodes were also asked to them. For each of the above question, multiple responses were given, and the patients were asked to give a "yes or no" response. Multiple responses were also accepted. Patients were interviewed only once during the during the period of data collection, even if they came for followup visits regularly. The data were analyzed by frequency and percentage.

Results : The study included 500 diabetic patients, more than half of the patients $(55.5 \%)$ of the study subjects were females. The mean age (standard deviation [SD]) of the study participants was $59.6( \pm 9.7)$ years and the age ranged from 29 to 85 years. Majority of the patients $(81 \%)$ were more than 50 years of age and were belonging to middle socioeconomic class (80\%). The mean (SD) duration of diabetes was $10.9( \pm 5.9)$ years with a duration ranging from 6 to 40 years (Table 1).

Table 1: Background characteristics of patients with Diabetes Mellitus $\mathrm{N}=500$

\begin{tabular}{|c|c|}
\hline Back Ground Characteristics & $f(\%)$ \\
\hline Age in Years & \\
1. Up to 50 & $95(19)$ \\
2. $51-60$ & $200(40)$ \\
3. > 60 & $205(41)$ \\
\hline Sex & $223(44.5)$ \\
1. Male & $277(55.5)$ \\
2. Female & \\
\hline Education & $280(56)$ \\
1. Illiterate & $220(44)$ \\
2. Literate & \\
\hline Monthly Income & $66(13)$ \\
1. Rs. $\leq 5000 /-$ & $34(7)$ \\
2. Rs. $5001-10,000 /-$ & $251(50)$ \\
3. Rs. $10,001-15,000 /-$ & $115(23)$ \\
4. Rs. $15,001-20,000 /-$ & $34(7)$ \\
5. Rs. $\geq 20.001$ & $76(15)$ \\
\hline Duration of Diabetes Mellitus & $230(46)$ \\
1. <5 years & $194(39)$ \\
2. 5 - 10 years
\end{tabular}


The common symptoms of hypoglycemia known to the study subjects were dizziness $(84.4 \%)$, weakness $(74.1 \%)$, and drowsiness (68.1\%). Headache (19.7\%) and seizures $(2.7 \%)$ as symptoms were known to less number of patients only (Table 2). Overall, 322 (64.4\%) diabetic patients had good knowledge on hypoglycemia (knowledge of at least three symptoms of hypoglycemia together with at least one precipitating factor and at least one remedial measure) and 56 (11.2\%) patients were not known even one symptom of hypoglycemia.

Table 2: Knowledge of symptoms of hypoglycemia among Diabetic patients $\mathrm{N}=500$

\begin{tabular}{|l|l|}
\hline \multicolumn{2}{|c|}{ Knowledge of Symptoms of Hypoglycemia } \\
\hline Symptoms * & $\mathrm{f}(\%)$ \\
\hline Dizziness & $422(84.4)$ \\
\hline Drowsiness & $341(68.1)$ \\
\hline Excessive hunger & $355(71)$ \\
\hline Sweating & $25(45)$ \\
\hline Seizures & $14(2.7)$ \\
\hline Head ache & $99(19.7)$ \\
\hline Weakness & $365(73)$ \\
\hline Loss of conscious ness & $195(39)$ \\
\hline confusion & $175(35)$ \\
\hline shaking & $165(33)$ \\
\hline *multiple response were allowed \\
\hline
\end{tabular}

More than two-thirds $(68.6 \%)$ of the patients knew that hypoglycemia may be precipitated by missing or delaying of meals. Exertion as a precipitating factor was known to $35.2 \%$ of patients (Table 3). At least one precipitating factor was known to 389 (77.8\%) patients and remedial measure, anyone, to be taken during an episode was known to 451 (90.2\%) of patients. Half of the patients (49.7\%) did not know even one complication of hypoglycemia.

Table3: Knowledge of precipitating factors of hypoglycemia and its remedial and preventive measures among Diabetic patients* $\mathrm{N}=500$
Jour of Diab and Endo Assoc of Nepal 2017; 1(1): 11-16

\begin{tabular}{|c|c|}
\hline \multicolumn{1}{|c|}{ Factors \& Measures } & $\mathbf{f ( \% )}$ \\
\hline Precipitating Factors & \\
1. Missing or delayed food & $343(68.6)$ \\
2. Exertion & $176(35.2)$ \\
3. Wrong dosage & $140(28)$ \\
4. Alcohol intake & $40(8)$ \\
\hline Complication of Hypoglycemia & $185(37)$ \\
1. Paralytic attack & $180(36)$ \\
2. Heart attack & $175(35)$ \\
3. Coma & $165(33)$ \\
4. Death & $75(15)$ \\
5. Fits & $245(49)$ \\
\hline Remedial measures during Hypoglycemia & $325(65)$ \\
1. Glucose powder or sugar & $195(39)$ \\
2. Go to casualty or emergency & $305(61)$ \\
3. department & \\
4. Dat food & $460(92)$ \\
\hline Preventive measures & $430(86)$ \\
1. Take timely meal & $240(48)$ \\
2. Take medication as adviced & - \\
3. Report to doctor & $370(74)$ \\
4. Adjust the medications & \\
5. Self-monitoring blood sugar & \\
\hline *multiple responses allowed &
\end{tabular}

Regarding management of hypoglycemia, $49 \%$ patients preferred taking glucose powder or sugar with water as an immediate measure. Majority of patients (65\%) felt going to the casualty or emergency department was the right option for hypoglycemia. Eating food which is either missed or delayed as a measure to be taken during an episode of hypoglycemia was known to $<40 \%$ of the study population.

Upon questioning their knowledge on prevention of further attacks, most of the patients (92\%) mentioned "taking timely meals" as a measure and $87 \%$, to take medications as per prescriptions of the doctor. Only a fifth of the patients were aware of "self-monitoring of blood glucose (SMBG) by glucometers" as a means to prevent further attacks as this will help them to identify hypoglycemia at an early stage, correlate with symptoms and take preventive measures (Table 3 ).

Thus, overall 331 (66.2\%) diabetic patients had knowledge of at least three symptoms of hypoglycemia together with at least one precipitating factor and at least one remedial measure and therefore were considered to have good knowledge on hypoglycemia. Higher age, illiteracy, low socioeconomic status was associated with poor knowledge whereas treatment with insulin along with oral hypoglycemic agents was associated with 
good knowledge on hypoglycemia. Sex and duration of disease were not associated with knowledge on hypoglycemia.

More than $50 \%$ of the patients attributed their knowledge of hypoglycemia to the doctor who treated them. A little lesser proportion (42.6\%) attributed their knowledge to their friends and relatives. A quarter learnt about hypoglycemia from the fellow patients who get treatment from the OP clinic (Figure 1).

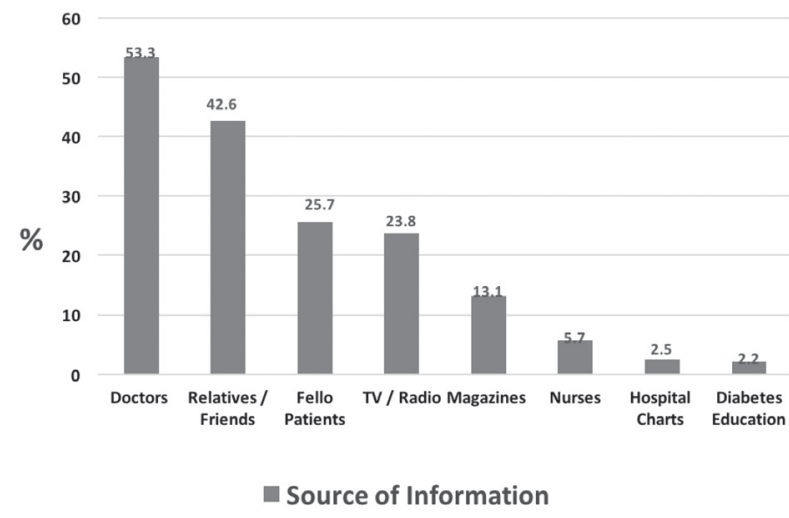

Figure 1:Source of knowledge on hypoglycemia among Diabetic Patients

\section{Discussion}

The study included 500 diabetic patients, more than half of the patients $(55.5 \%)$ of the study subjects were females. The mean age (SD) of the study participants was 59.6 ( \pm 9.7 ) years and the age ranged from 29 to 85 years. Majority of the patients (81\%) were more than 50 years of age and were belonging to middle socioeconomic class (80\%). The mean (SD) duration of diabetes was 10.9 (5.9) years with a duration ranging from 6 to 40 years. Higher age, illiteracy, and low socioeconomic status were associated with poor knowledge whereas treatment with insulin along with OHAs was associated with good knowledge on hypoglycemia. Sex and duration of disease were not associated with knowledge on hypoglycemia.

Diabetes is fast gaining the status of a potential epidemic in India with more than 62 million diabetics. According to Wild et al the prevalence of diabetes is predicted to double globally from 171 million in 2000 to 366 million in 2030 with a maximum increase in India. It is predicted that by 2030 diabetes mellitus may afflict up to 79.4 million individuals in India.
With respect to the knowledge on symptoms of hypoglycemia, dizziness, weakness, and drowsiness were the ones known to most patients and headache and seizures, the least. Hypoglycemia may manifest with any symptom, and common symptoms may not always be the first to appear. Therefore, the patients must be aware of every symptom in order to recognize early and take immediate corrective measures.

Missing meals as a precipitating factor was known to two-thirds of the patients and exertion to a third of the patients only. It is very important that the patients are well aware of the precipitating factors/causes so that they may take appropriate precautions such as taking toffees or packed food before planning to travel or exercise. Only a third of the patients had knowledge on the complications, and $<50 \%$ knew that they have to report such episodes to the doctor. Awareness about the complications would induce the patients to take this condition seriously and report any such episodes to the physician immediately.

Even though $85 \%$ of the study population are diabetic for more than 5 years, Self-Monitoring of Blood Glucose (SMBG) as a way to prevent future episodes was known to a fifth of the patients only. It is well-known that SMBG aids in better glycemic control and prevention of hypoglycemia in diabetes patients by allowing for adjustments in diet, physical activity, and pharmacotherapy in response to test results. , In this study, the knowledge on hypoglycemia decreased as age increased. This finding is shown in other studies too. This might have been because of age-related cognitive decline insisting the importance of periodical educational programs to reinforce their knowledge.

Among the participants $49 \%$ preferred taking glucose powder or sugar dissolved in water as an immediate measure for their hypoglycemic symptoms. Majority (65\%) felt going to hospital emergency department was the right option for immediate management of Hypoglycemia. The more literate the patient was, he could identify symptoms of hypoglycemia and take corrective measures to avoid severe hypoglycemia. A study conducted in England among Type-2 diabetes, $5.9 \%$ patients on 
OHA's in the age group of 40 to 65 years experienced hypoglycemic symptoms. None of these patients required hospitalization as they aware on the immediate management of hypoglycemia as they received good diabetic education. Ya-Chun et al studied 1195 patients with type 2 DM and observed $7.4 \%$ to have experienced severe hypoglycemia. Patients on insulin experienced hypoglycemia more frequently (17.8\%) as compared to patients on OHA's (6.3\%). The risk factors for developing severe hypoglycemia were older age, lower literacy level and insulin therapy. Zammitt NN and Frier BM observed an increased risk of hypoglycemia among older adults and those with diabetes for many years.

The immediate treatment of hypoglycemia should be known by all the diabetic patients, so that need for hospitalization could be avoided. Illiterate patients and elderly patients with dementia must be more educated about hypoglycemia. Thus improving patient skills selfmanagement, self-monitoring of sugar and adjustments of dose based on requirements can reduce the risk of hypoglycemia. Severe hypoglycemia is usually associated with increased mortality, impaired cognitive function and affects patient's quality of life. Frequent hypoglycemic spells can burden the existing healthcare facilities and productivity at workplace can be affected.

This study emphasizes the role of health care workers-doctors, nurses, diabetes educators, lab technicians in providing health education to the patients during every visit. They must educate on target levels, importance of SMBG, symptoms of hypoglycemia, the ways of preventing it and immediate remedial measures to be adopted during every visit. Furthermore, periodical educational reinforcement programs need to be conducted for all diabetic patients which must include topics on hypoglycemia.

This is one of the few studies in India to study exclusively about the knowledge on hypoglycemia in detail among the type 2 diabetic patients. In India, which has the second largest number of diabetes patients, there is negligible data on the epidemiology of hypoglycemia. The limitation of the study is that the knowledge is assessed among patients attending a diabetes free Clinic in a tertiary care hospital and may not be accurately representing that in a community comprising both affordable and poor patients.

\section{Conclusions}

Although the knowledge on the symptoms, remedial measures, and prevention of hypoglycemic episodes was good among the type 2 diabetic patients in the study, there were gap in knowledge on important aspects like precipitating factors, target levels etc., which need to be addressed by health care workers through regular educational programs.

\section{References:}

1. Kalra S, Mukherjee JJ, Venkataraman S, Bantwal G, Shaikh S, Saboo B, et al. Hypoglycemia: The neglected complication. Indian J Endocrinol Metab. 2013;17:819-34.

2. Patel A, MacMahon S, Chalmers J, Neal B, Billot L, et al. ADVANCE Collaborative Group. Intensive blood glucose control and vascular outcomes in patients with type 2 diabetes. $N$ Engl J Med. 2008;12(358):2560-72.

3. Duckworth W, Abraira C, Moritz T, Reda D, Emanuele N, Reaven PD, et al. Glucose control and vascular complications in veterans with type 2 diabetes. N Engl J Med. 2009;360:129-39.

4. American Diabetes Association. Standards of medical care in diabetes-2014. Diabetes Care. 2014;37(Suppl 1):S14-80.

5. American Diabetes Association. Standards of medical care in diabetes-2014. Diabetes Care. 2014;37(Suppl 1):S14-80.

6. Wild S, Roglic G, Green A, Sicree R, King H. Global prevalence of diabetes-estimates for the year 2000 and projections for 2030. Diabetes Care 2004; 27:1047-53.

7. Kaveeshwar SA, Cornwall J. The current state of diabetes mellitus in India. The Australasian Med J $2014 ; 7: 45$.

8. Cefalu CA, Cefalu WT. Controlling hypoglycemia in type 2 diabetes: Which agent for which patient? J Fam Pract. 2005;54:855-62.

9. Benjamin EM. Self-monitoring of blood glucose: The basics. Clin Diabetes. 2002;20:45-7.

10. Garg S, Hirsch IB. Self-monitoring of blood glucose. Int J Clin Pract Suppl. 2010;166:1-10.

11. Thomson FJ, Masson EA, Leeming JT, Boulton AJ. Lack of knowledge of symptoms of hypoglycaemia by elderly diabetic patients. Age Ageing. 1991;20:404-6.

12. Mutch WJ, Dingwall-Fordyce I. Is it a hypo? Knowledge of the symptoms of hypoglycaemia in elderly diabetic patients. Diabet Med. 1985;2:54-6.

13. Al-Adsani AM, Moussa MA, Al-Jasem LI, Abdella 
NA, Al-Hamad NM. The level and determinants of diabetes knowledge in Kuwaiti adults with type 2 diabetes. Diabetes Metab. 2009;35:121-8.

14. Jennings AM, Wilson RM, Ward JD. Symptomatic hypoglycemia in NIDDM patients treated with oral hypoglycemic agents. Diabetes Care 1989; 12: 203-8.

15. Hsiao YC, Chien MN. Severe hypoglycemia in type 2 diabetes a hospital-based retrospective study. J Intern Med Taiwan 2006; 17: 73-7.

16. Zammitt NN, Frier BM. Hypoglycemia in type 2 diabetes: pathophysiology, frequency, and effects of different treatment modalities. Diabetes Care 2005; 28:2948-61.
17. Morales J, Schneider D. Hypoglycemia. The American Journal of Medicine 2014; 127: S17-S24.

18. Viswanathan $M$, Joshi SR, Bhansali A. Hypoglycemia in type 2 diabetes: Standpoint of an experts' committee (India hypoglycemia study group) Indian J Endocrinol Metab. 2012;16:894-8. 